\title{
Comparison of Two Multisheet Transmission Windows for Millimeter-Wave Radiometers
}

\author{
MARK D. JACOBSON, MEMBER, IEEE, JACK B. SNIDER, MEMBER, IEEE, AND DAVID C. HOGG, FELlOW, IEEE
}

\begin{abstract}
The design and performance of a triple-sheet Mylar window and a Teflon and foam window are studied theoretically and experimentally to determine which window produces the smaller brightness temperature variations and, therefore, leads to better accuracy of radiometers operating at frequencies of $20-90 \mathrm{GHz}$. Theoretical brightness temperatures due to emission and reflection by the windows are calculated and compared, and experimental measurements obtained by using the clear atmosphere as a radiation source are compared. Both theory and experiment indicate that the Teflon and foam window produces smaller brightness temperature variations at these operating frequencies. Effects of the windows on integrated water vapor and liquid measurements are also examined; errors are significant for the triple-sheet Mylar window but are negligible for the Teflon and foam window. It is observed that use of the Teflon and foam design results in acceptable small errors in radiometric measurements of water vapor and cloud liquid.
\end{abstract}

\section{INTRODUCTION}

$\mathrm{F}$

OR THE PAST several years the National Oceanic and Atmospheric Administration (NOAA) has successfully operated a dual-channel $(20.6$ and $31.6 \mathrm{GHz})$ steerable radiometer in the measurement of water vapor and cloud liquid. A shielded triple-sheet Mylar microwave window [1] has been used to provide operation under all-weather conditions. Water vapor is determined primarily by measurement of the brightness temperature from emission at $20.6 \mathrm{GHz}$ and the amount of liquid water contained in clouds primarily by emission at $31.6 \mathrm{GHz}$. Recently, a third radiometric channel operating at $90.0 \mathrm{GHz}$ was installed with the dual-channel radiometer. The additional channel was added chiefly to increase the sensitivity to liquid water in clouds (often supercooled liquid). The goal is to find a window that is satisfactory from an applications point of view, at $90.0 \mathrm{GHz}$ along with 20.6 and $31.6 \mathrm{GHz}$ as well as frequencies in the $50 \mathrm{GHz}$ band. Fig. 1 shows a cross section of the triple-channel steerable instrument. A heat pump and air conditioner (both not shown) are used to stabilize the temperature of the insulated trailer housing. The azimuth flat and bearing are protected with a weather-tight cover fitted with the recessed triple-sheet window. The window is formed of three Mylar sheets $0.05 \mathrm{~mm}$ thick, each separated by an air gap of about $50 \mathrm{~mm}$. Mylar was chosen initially because of its mechanical strength and

Manuscript received February 24, 1987; revised August 14, 1987

M. D. Jacobson and J. B. Snider are with the Wave Propagation Laboratory, NOAA/Environmental Research Laboratories, Boulder, CO 80303.

D. C. Hogg is with the Cooperative Institute for Research in Environmenta Sciences, University of Colorado, Boulder, CO 80309.

IEEE Log Number 8719168

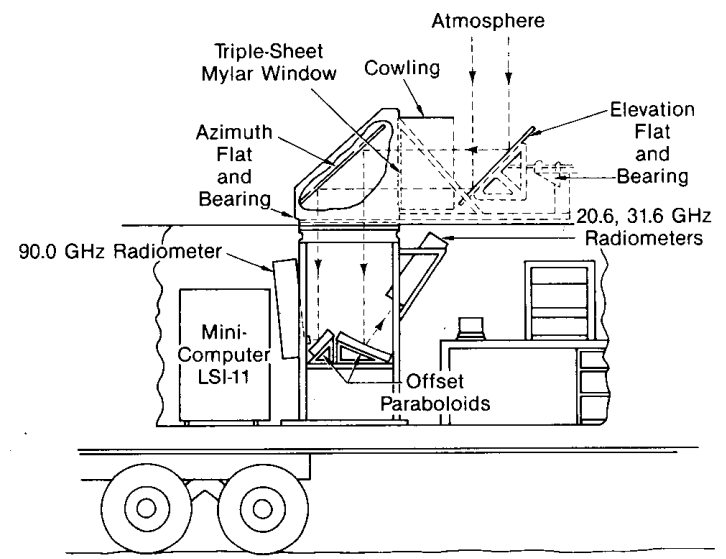

Fig. 1. Side view of steerable triple-channel radiometer; path of central ray from the zenith is shown as dashed lines with arrows. These radiometers emit about $318 \mathrm{~K}$ (reference load temperature) when in antenna mode (i.e., viewing atmosphere).

satisfactory electrical properties at 20.6 and $31.6 \mathrm{GHz}$. The triple-sheet window helps prevent condensation on the outer surface during warm humid conditions, protects the radiometers from the weather, and helps maintain a constant ambient temperature for the millimeter-wave and electronic components. An external heater and blower on the floor of the cowling can be added if condensation on the outer sheet is still objectionable.

\section{Window CONSIDERATIONS}

A transmission window modifies the transiting wavefronts through absorption, reflection, refraction, and scattering. These undesirable modifications are a function of the window material and thickness. Radiometric instruments that can detect noise changes of less than $1 \mathrm{~K}$ are especially sensitive to the effects of transmission windows. For ground-based radiometric instruments operating under all-weather conditions multisheet windows (dielectric sheets separated by air gaps) are used instead of single windows (one dielectric sheet) in part because they prevent condensation on the outer surface during warm humid conditions, thus avoiding the undesirable effects of a wetted window [2]. The rationale is that a multisheet window provides good thermal insulation. To produce the smallest possible distortion of the incoming radiation, the thickness and dielectric properties of the sheets are chosen to be as small as possible. However, the thin 
dielectric sheets are flexible, and when winds or other forces impinge upon a multisheet window the spacing between adjacent sheets may vary, thereby inducing changes in the transmission (and reflection) behavior. This sheet movement is analogous to a varying air-gap spacing which creates a fluctuating energy reflection because of interference. Minimization of the energy fluctuations is a critical factor in the design.

Accurate brightness temperature measurements of the atmosphere are necessary to determine precisely the amounts of water vapor and liquid water in the atmosphere along the antenna beam. The observed brightnesses, expressed as absorption values, are combined in simultaneous equations to yield independently the amounts of liquid water and water vapor within the antenna beam. Equations for calculating the vapor and liquid are given in Section III. An absolute brightness temperature measurement is obtained by properly calibrating the instrument, using what is commonly called a tipping curve calibration [1]. These measurements are made on cloudless days when the emission from the atmosphere is unchanging. By scanning the elevation, absorption measurements can be plotted against the number of air masses (linear versus secant of the zenith angle). This calibration accounts for losses and emissions between the elevation flat and the radiometer detector output. Thus the effects of a multisheet window on the incoming radiation are taken into account for the mean air gap thickness existing during the calibration. However, when the dielectric sheets begin to move (a wind can cause this), the air-gap thickness varies, thereby inducing changes in the electromagnetic interaction behavior between the sheets. The variation may produce significant differences between the measured and actual sky brightness temperatures. This fluctuating energy is of concern because it can constitute a significant source of contamination of the brightness temperature measurements (as does a wet reflector [3] and/or window).

Since the required accuracy of the triple-channel radiometer is less than $0.5 \mathrm{~K}$, it is necessary to minimize the brightness temperature variations (peak-to-peak) produced by the multisheet window to within a few tenths of a Kelvin. To determine which produces the smaller brightness temperature variations, two multisheet windows are considered: the triplesheet Mylar window and a Teflon and foam window. To the best of our knowledge, the Teflon and foam window was first implemented by James R. Jordan of the Wave Propagation Laboratory.

\section{TheORY AND COMPUTATION}

A simple but realistic model is used to determine if either of the multisheet windows will produce no more than a few tenths of a Kelvin peak-to-peak brightness temperature variation at 20.6, 31.6, and 90.0 Ghz. The observed brightness temperature $T_{b}$ at the offset paraboloid (Fig. 1) is the sum of the transmitted atmospheric brightness temperature $\mathrm{TT}_{a}$ (where T is the power transmission of the multisheet window), the brightness temperature from the multisheet window $T_{\mathrm{bw}}$, and the reflected reference load temperature $R_{r} T_{r}$ (where $R_{r}$ is the power reflection of the multisheet window as viewed from the paraboloid). Thus

$$
T_{b}=\mathrm{TT}_{a}+T_{\mathrm{bw}}+R_{r} T_{r}
$$

where $T_{a}$ is the atmospheric brightness temperature and $T_{r}$ the thermodynamic temperature of the waveguide reference load.

From Kirchoff's law and by conservation of energy, the brightness temperature of the multisheet window is given by

$$
T_{\mathrm{bw}}=\left(1-T-R_{a}\right) T_{w}
$$

where $T_{w}$ is the thermodynamic temperature of the multisheet window $(\mathrm{K})$ and $R_{a}$ the power reflection of the multisheet window as viewed from the atmosphere. Substituting (2) into (1),

$$
T_{b}=\mathrm{TT}_{a}+\left(1-T-R_{a}\right) T_{w}+R_{r} T_{r} .
$$

The atmospheric brightness temperature $T_{a}$ is measured by the radiometer and the temperature of the multisheet window $T_{w}$ and the temperature of the reference load $T_{r}$ by thermistors.

To obtain an estimate of the power reflections $R_{a}$ and $R_{r}$ and power transmission $T$, we deal with the simplest possible model: an ideal antenna with no sidelobes and smooth dielectric (window) sheets of uniform thickness of infinite extent, separated by a uniform air-gap thickness. In addition, we employ mononchromatic frequencies at normal incidence. A full-wave calculation is necessary to include irregular sheet thickness, irregular air gap thickness, finite sheet area, and finite bandwidth, but the improved accuracy over the simple model for this application is not enough to justify its use. Thus a transmission line equivalent circuit for the multisheet window can be used (see Fig. 2) to calculate $R_{a}, R_{r}$, and $T$. The propagation constants and characteristic impedances of the different media are defined in [4]:

$$
\gamma=i 2 \pi f \sqrt{\mu_{0}\left(\epsilon^{\prime}-i \epsilon^{\prime \prime}\right)}, \quad \text { propagation constant }\left(m^{-1}\right)
$$

$$
z=\sqrt{\frac{\mu_{0}}{\epsilon^{\prime}-i \epsilon^{\prime \prime}}}, \quad \text { characteristic impedance }(\Omega)
$$

where

$$
\begin{aligned}
& i=\sqrt{-1}, \\
& f \quad \text { frequency under consideration (Hz), } \\
& \mu_{0}=4 \pi \times 10^{-7}, \text { free-space permeability }(\mathrm{H} / \mathrm{m}), \\
& \epsilon^{\prime}=\epsilon_{r} / \mu_{0} c^{2},(\mathrm{~F} / \mathrm{m}), \\
& \epsilon_{r} \quad \text { relative permittivity (dimensionless), } \\
& c \quad \sim 3 \times 10^{8}, \text { speed of light in a vacuum }(\mathrm{m} / \mathrm{s}), \\
& \epsilon^{\prime \prime}=\epsilon^{\prime} \tan \delta(\mathrm{F} / \mathrm{m}), \\
& \tan \delta \quad \text { loss tangent (dimensionless). }
\end{aligned}
$$

To facilitate the use of the transmission line analogy in Fig. 2 , the multisheet two-port network (dielectric sheet 1-air gapdielectric sheet 2-air gap- $\cdots$-dielectric sheet $n$-1-air gapdielectric sheet $n$ ) is cascaded into an equivalent network. Computational effort in the analysis of cascaded two ports, as in Fig. 2, can be simplified using the $A B C D$ matrix representation [5]. The elements in this matrix are defined in terms of voltages and currents at the two ports of the desired 


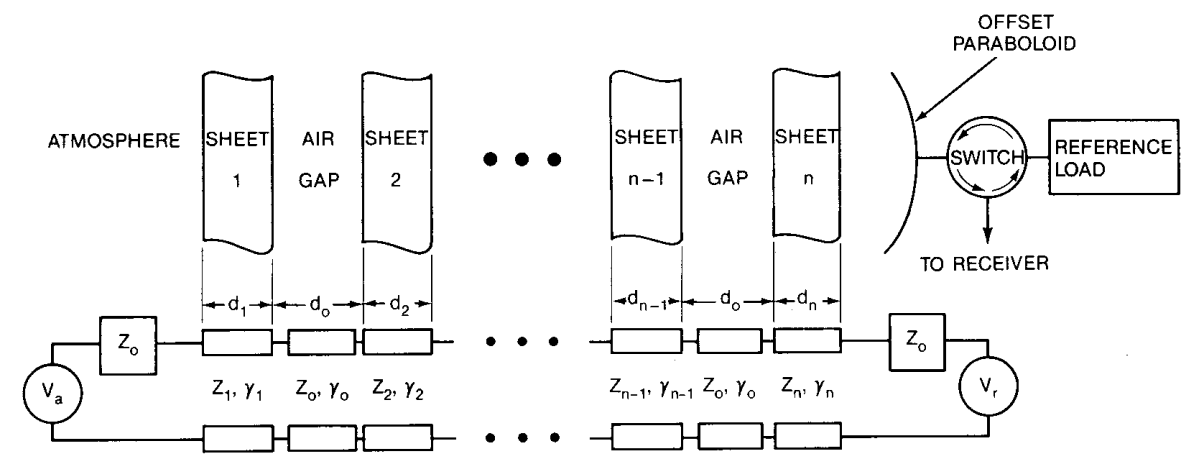

Fig. 2. Transmission line equivalent circuit for multisheet window. Propagation constants and characteristic impedances are given by $\gamma_{0}, \gamma_{1}, \gamma_{2}, \cdots, \gamma_{n-1}, \gamma_{n}$, and $Z_{0}, Z_{1}, Z_{2}, \cdots, Z_{n-1}, Z_{n}$, respectively. Atmospheric and reference load radiation sources are $V_{a}$ and $V_{r}$, respectively.

network. The $A B C D$ matrix is especially suited for cascading several two-port networks and is obtained by multiplying the individual $A B C D$ matrices together in the proper order. For Fig. 2 this is readily shown to be

$$
\begin{aligned}
{\left[\begin{array}{cc}
A & B \\
C & D
\end{array}\right]=} & {\left[\begin{array}{cc}
C_{1} & Z_{1} S_{1} \\
\frac{S_{1}}{Z_{1}} & C_{1}
\end{array}\right]\left[\begin{array}{cc}
C_{0} & Z_{0} S_{0} \\
\frac{S_{0}}{Z_{0}} & C_{0}
\end{array}\right]\left[\begin{array}{cc}
C_{2} & Z_{2} S_{2} \\
\frac{S_{2}}{Z_{2}} & C_{2}
\end{array}\right] } \\
& \cdot\left[\begin{array}{cc}
C_{0} & Z_{0} S_{0} \\
\frac{S_{0}}{Z_{0}} & C_{0}
\end{array}\right] \cdots\left[\begin{array}{cc}
C_{n-1} & Z_{n-1} S_{n-1} \\
\frac{S_{n-1}}{Z_{n-1}} & C_{n-1}
\end{array}\right] \\
& \cdot\left[\begin{array}{cc}
C_{0} & Z_{0} S_{0} \\
\frac{S_{0}}{Z_{0}} & C_{0}
\end{array}\right]\left[\begin{array}{ll}
C_{n} & Z_{n} S_{n} \\
\frac{S_{n}}{Z_{n}} & C_{n}
\end{array}\right]
\end{aligned}
$$

where

$C_{i}=\cosh \left(\gamma_{i} d_{i}\right)$

$S_{i}=\sinh \left(\gamma_{i} d_{i}\right)$,

$Z_{i} \quad$ impedance of medium $i(\Omega)$,

$d_{i} \quad$ medium $i$ thickness $(\mathrm{m})$,

$\gamma_{i} \quad$ propagation constant in medium $i\left(\mathrm{~m}^{-1}\right)$,

for $i=0$ : air gap,

$$
\begin{aligned}
& 1 \text { : dielectric sheet } 1 \text {, } \\
& 2 \text { : dielectric sheet } 2 \text {, } \\
& \begin{array}{ccc}
\vdots & \vdots & \vdots
\end{array}
\end{aligned}
$$

To obtain the amplitude reflections $\left(r_{a}\right.$ and $\left.r_{r}\right)$ and transmission $(t)$, we convert the overall $A B C D$ matrix in (6) to a scattering matrix; for a reciprocal nework this gives [5]

$$
\begin{gathered}
r_{a}=\frac{A Z_{0}+B-C Z_{0}^{2}-D Z_{0}}{G} \\
r_{r}=\frac{-A Z_{0}+B-C Z_{0}^{2}+D Z_{0}}{G}
\end{gathered}
$$

TABLE I

Electrical Properties of MYlar [6], Teflon [7], [8], AND LowLOSS FOAM AT 20.6, 31.6, AND $90.0 \mathrm{GHZ}$

\begin{tabular}{cccc}
\hline $\begin{array}{c}\text { Frequency } \\
\text { (GHz) }\end{array}$ & Material & $\epsilon_{r}$ & $\tan \delta \times 10^{-4}$ \\
\hline \multirow{4}{*}{20.6} & Mylar & 3.0 & 50 \\
& Teflon & 2.08 & 6 \\
& Foam & 1.03 & 1 \\
31.6 & Mylar & 3.0 & 50 \\
& Teflon & 1.99 & 2 \\
& Foam & 1.03 & 1 \\
90.0 & Mylar & 3.0 & 50 \\
& Teflon & 2.06 & 2 \\
& Foam & 1.03 & 2 \\
\hline
\end{tabular}

$$
t=\frac{2 Z_{0}}{G}
$$

where

$$
G=A Z_{0}+B+C Z_{0}^{2}+D Z_{0}
$$

The power reflections and transmission are

$$
\begin{gathered}
R_{a}=\left|r_{a}\right|^{2} \\
R_{r}=\left|r_{r}\right|^{2} \\
T=|t|^{2} .
\end{gathered}
$$

The magnitudes of (10), (11), and (12) are obtained from the approximate values of dielectric constant and loss tangent for Mylar, Teflon, and low-loss foam given in Table I for the three frequencies of interest. An approximate window temperature of $298 \mathrm{~K}$ is used (see discussion of the controlled experiment in Section IV). Given these constants, theoretical and experimental results can be compared. The dielectric properties of Mylar are only approximately known above 10 $\mathrm{GHz}$ (the last published data point we located was at $8.6 \mathrm{GHz}$ [7]). Even though the foam has been tested only up to $10 \mathrm{GHz}$, its dielectric properties are assumed not to change significantly through $90 \mathrm{GHz}$, as is shown in Table I. Teflon, on the other hand, has been tested to $100 \mathrm{GHz}$, and its dielectric properties are apparently known accurately for the frequencies of 
interest. The low-loss foam used (ECCOFOAM PP-2) is manufactured by Emerson and Cuming, Inc.

To determine the effect of the multisheet window on the atmospheric brightness temperature, the atmospheric brightness temperature is subtracted from (3), giving the "difference temperature",

$$
T_{d}=(T-1) T_{a}+\left(1-T-R_{a}\right) T_{w}+R_{r} T_{r} .
$$

This brightness temperature difference gives the contribution of the multisheet window to the apparent sky brightness temperature. $T_{d}=0 \mathrm{~K}$ when the multisheet window is removed. A positive $T_{d}$ indicates that additional energy is received at the paraboloid because of reflection by and emission from the window.

Recovery of the amounts of atmospheric water vapor and liquid water from trifrequency radiometer measurements involves a statistical inversion [1], [9], [10]. Application of this procedure results in the following expressions for integrated water vapor $V(\mathrm{~cm})$ and liquid water $L(\mathrm{~cm})$ :

$$
\begin{aligned}
V=C_{0 V}+C_{1 V} T_{\mathrm{dry}}+C_{2 V} P & +C_{3 V} \mathrm{RH} \\
& +C_{20 V} \tau_{20}+C_{31 V} \tau_{31}+C_{90 V} \tau_{90} \\
L=C_{0 L}+C_{1 L} T_{\mathrm{dry}}+C_{2 L} P & +C_{3 L} \mathrm{RH} \\
& +C_{20 L} \tau_{20}+C_{31 L} \tau_{31}+C_{90 L} \tau_{90}
\end{aligned}
$$

where $T_{\mathrm{dry}}, P$, and $\mathrm{RH}$ are the physical temperature $(\mathrm{K})$, pressure $(\mathrm{mb})$, and relative humidity, respectively, at the earth's surface, and the C's are the retrieval coefficients calculated from a multiyear sample of radiosonde observations made at or near the location where the radiometric measurements are to be made.

In (14) and (15), $\tau_{20}, \tau_{31}$, and $\tau_{90}$ are the total atmospheric absorptions; these are computed from the brightness temperatures $T_{b(f)}$, measured by the radiometer using the relationship [1]

$$
\tau_{(f)}=-\ln \left(\frac{T_{m r(f)}-T_{b(f)}}{T_{m r(f)}-T_{b b}}\right)
$$

where $T_{m r(f)}$ is an effective atmospheric radiating temperature at each operating frequency $f, T_{b b}$ is the cosmic background temperature $(2.9 \mathrm{~K})$, and the absorption $\tau_{(f)}$ is in nepers. It is customary to calculate the $T_{m r}$ values in (16) from a multiyear sample of radiosonde data measured at or near the location where the radiometric measurements are to be made.

To determine the effect of the multisheet window on the measurements of water vapor and liquid, the differences between the water vapor and liquid measurements with and without the window are taken:

$$
\begin{aligned}
& V_{d}=V_{\text {(with window) }}-V_{\text {(without window) }} \\
& L_{d}=L_{\text {(with window) }}-L_{\text {(without window) }} .
\end{aligned}
$$

Calculated plots of brightness temperature difference $T_{d}$, water vapor difference $V_{d}$, and liquid difference $L_{d}$ versus airgap thickness for the Teflon and foam window are shown in Fig. 3; similar curves exist for the triple-sheet Mylar window.
These curves were calculated from (3), (13), (17), and (18), and several values of the atmospheric brightness temperatures $T_{a}$ were used. The window temperature $T_{w}$ and the reference load temperature $T_{r}$ are fixed at 298 and $318 \mathrm{~K}$, respectively, for all five graphs. The Teflon and foam window consists of one Teflon sheet $0.05 \mathrm{~mm}$ thick and one low-loss foam sheet $6.4 \mathrm{~mm}$ thick (thinnest available) separated by a $50-\mathrm{mm}$ air gap. The column labeled "theory" in Table II gives the theoretical mean and peak-to-peak values for brightness temperature difference $T_{d}$ for the two types of windows. As mentioned in Section II, the maximum variation in $T_{d}$ for a particular window should be on the order of a few tenths of a Kelvin to permit accurate brightness temperature measurements of the atmosphere. Based on the theoretical values in Table II, the triple-sheet Mylar window performance is unsatisfactory at all three frequencies (i.e., $T_{d}$ (peak to peak) is greater than a few tenths of a Kelvin), and the mean differences are quite large. On the other hand, the Teflon and foam window performs well at these three frequencies (i.e., $T_{d}$ (peak to peak) is on the order of a few tenths of a Kelvin) and the mean difference is small. Computed variations (peak to peak) in water vapor $V_{d}$ and liquid water $L_{d}$ produced by the Teflon and foam window are negligible (see ordinates of Fig. 3(d) and (e)) since these values are near or below the noise level of the radiometer but corresponding variations (peak to peak) of about 3 and $0.01 \mathrm{~mm}$, respectively, are produced by the triple-sheet Mylar window. The measured values of $T_{d}$, $V_{d}$, and $L_{d}$ are discussed in Section IV.

The different characteristics of the Teflon and foam sheets are the key factors that minimize the unwanted noise fluctuations. The first-order explanation for this minimization is that the brightness temperature caused by the Teflon sheet is due mainly to reflections at the air-Teflon interfaces (because the relative permittivity is fairly high) and not by emission (because the loss tangent is small). On the other hand, the brightness of the foam sheet is caused mainly by the absorption in the foam (the sheet is fairly thick) and not by reflection (because the relative permittivity is near unity). Because of these different characteristics, little energy interaction occurs between the two sheets, which means that a minimal fluctuating brightness temperature will be set up by the window. Table III lists relative absorption (emission) and reflection of the Teflon and foam window at the three frequencies of interest.

\section{Measurements}

On August 15, 1986, the NOAA steerable triple-channel radiometer was used to test the theory by viewing in the zenith direction with and without both types of window. Weather conditions had a critical role in the experiment, i.e., cloudless skies were required to ensure that the atmospheric zenith brightness temperature would remain fairly constant during the measurements. To obtain a fluctuating energy pattern, the window frame was physically shaken to simulate a typical windy condition. Fig. 4 is a photograph of the Teflon and foam window used in this experiment; the triple-sheet Mylar window is similar in appearance.

Before the brightness temperature on the two windows was measured, three tipping curve calibrations were performed 


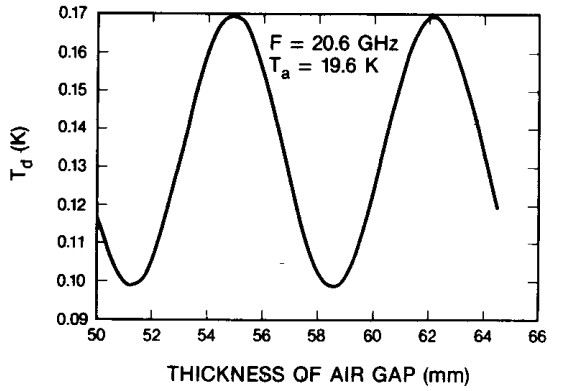

(a)

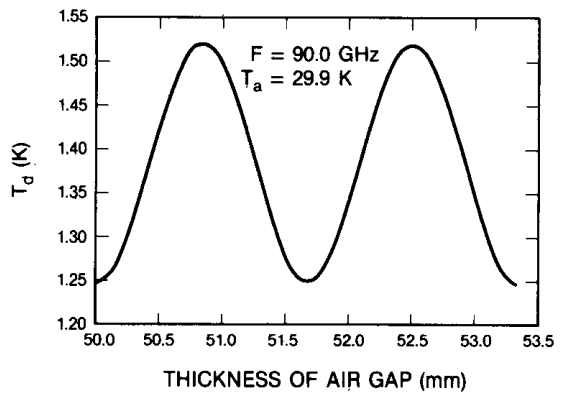

(c)

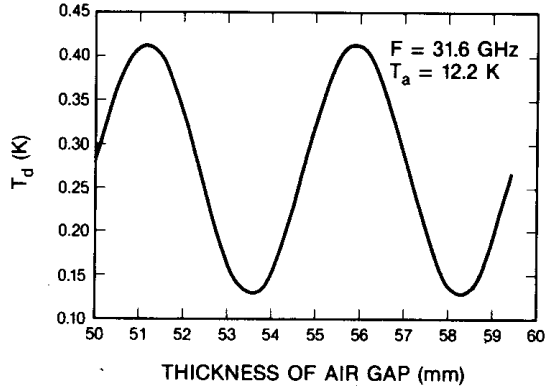

(b)

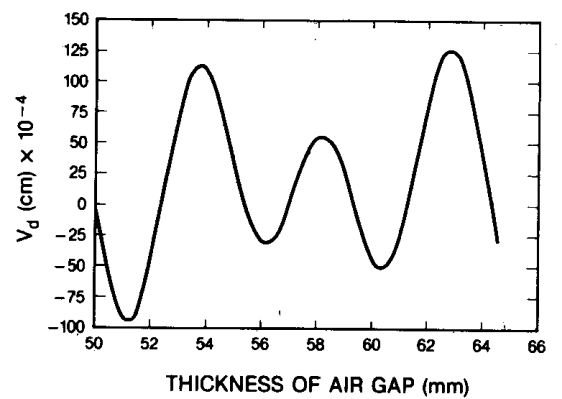

(d)

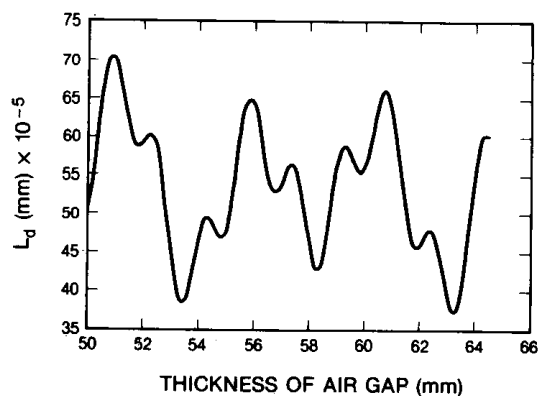

(e)

Fig. 3. Computed (a)-(c) brightness temperature difference $T_{d}$, (d) apparent water vapor difference $V_{d}$, and (e) liquid water difference $L_{d}$ values versus air gap thickness for Teflon and foam window with $d_{1}=0.05 \mathrm{~mm}$ (Teflon) and $d_{2}=16.4 \mathrm{~mm}$ (foam). $T_{w}$ and $T_{r}$ are fixed at 298 and $318 \mathrm{~K}$, respectively, for all five graphs.

TABLE II

THEORETICAL AND EXPERIMENTAL BRIGHTNESS TEMPERATURES FOR THE TWO MULTISHEET WINDOWS $\left(T_{w}\right.$ AND $T_{r}$ ARE FIXED AS MEASURED AT 298 AND $318 \mathrm{~K}$, RESPECTIVELY); USING THE PRESENT TECHNIQUE, PEAK-TO-PEAK FLUCTUATIONS WERE NOT MEASURABLE AT 20.6 AND $31.6 \mathrm{GHz}$

\begin{tabular}{|c|c|c|c|c|c|}
\hline \multirow[b]{2}{*}{$\begin{array}{c}\text { Frequency } \\
\text { (GHz) }\end{array}$} & \multirow[b]{2}{*}{ Window } & \multicolumn{2}{|c|}{$\begin{array}{c}\text { Peak-to-Peak } \\
T_{d}\end{array}$} & \multicolumn{2}{|c|}{$\begin{array}{c}\text { Mean } \\
T_{d}\end{array}$} \\
\hline & & $\begin{array}{c}\text { Theory } \\
\text { (K) }\end{array}$ & $\begin{array}{c}\text { Measure } \\
\text { (K) }\end{array}$ & $\begin{array}{c}\text { Theory } \\
\text { (K) }\end{array}$ & $\begin{array}{c}\text { Measure } \\
\text { (K) }\end{array}$ \\
\hline \multirow{3}{*}{20.6} & Mylar & 1.3 & na & 0.9 & 1.5 \\
\hline & Teflon/foam & 0.1 & na & 0.1 & 0.0 \\
\hline & Mylar & 3.0 & na & 1.9 & 3.1 \\
\hline \multirow[t]{2}{*}{31.6} & Teflon/foam & 0.3 & na & 0.3 & 0.2 \\
\hline & Mylar & 21.7 & 6.4 & 11.9 & 15.2 \\
\hline 90.0 & Teflon/foam & 0.3 & 0.5 & 1.4 & 0.6 \\
\hline
\end{tabular}

TABLE III

EMISSION AND REFLECTION FROM A TEFLON AND FOAM WINDOW AT 20.6, 31.6, AND $90.0 \mathrm{GHZ}\left(T_{w}\right.$ AND $T_{r}$ ARE FIXED AT 298 AND $318 \mathrm{~K}$ RESPECTIVELY)

\begin{tabular}{|c|c|c|c|c|}
\hline \multirow[b]{2}{*}{$\begin{array}{c}\text { Frequency } \\
(\mathrm{GHz})\end{array}$} & \multicolumn{2}{|c|}{$\begin{array}{c}\text { Power } \\
\text { Emission }\end{array}$} & \multicolumn{2}{|c|}{$\begin{array}{c}\text { Power } \\
\text { Reflection }\end{array}$} \\
\hline & $\begin{array}{l}\text { Teflon } \\
\text { (K) }\end{array}$ & $\begin{array}{l}\text { Foam } \\
(\mathrm{K})\end{array}$ & $\begin{array}{c}\text { Teflon } \\
\text { (K) }\end{array}$ & $\begin{array}{c}\text { Foam } \\
\text { (K) }\end{array}$ \\
\hline 20.6 & 0.01 & 0.08 & 0.04 & 0.01 \\
\hline 31.6 & 0.00 & 0.13 & 0.09 & 0.06 \\
\hline 90.0 & 0.01 & 0.73 & 0.79 & 0.01 \\
\hline
\end{tabular}




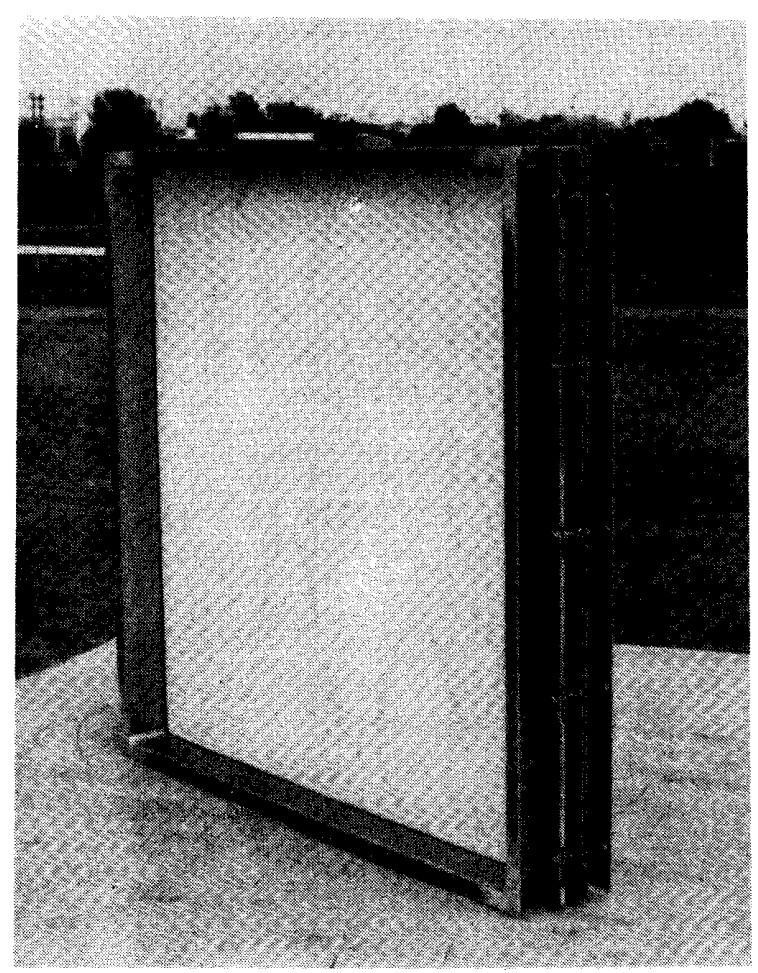

Fig. 4. Teflon and foam window.

with the triple-sheet Mylar window, and Teflon and foam window, and no window in place. These calibrations allow for precise brightness temperature measurements for each type of window. With no window present the brightness temperature of the atmosphere, $T_{a}$, alone is measured; thus the brightness temperature caused by a multisheet window can then be found by comparison.

The data were collected in the following way: with no window present the atmospheric zenith brightness temperatures at $20.6,31.6$, and $90.0 \mathrm{GHz}$ were measured for about 5 min. Then the Teflon and foam window was inserted and physically shaken for about $5 \mathrm{~min}$; during this time the zenith brightness temperatures were measured. Next the triple-sheet Mylar window was inserted and shaken for about $5 \mathrm{~min}$, and the zenith brightness temperatures measured. This process was repeated several times (the data are shown in Fig. 5); the unprocessed data were written on a removable magnetic disk every $5 \mathrm{~s}$. The data consist of average values obtained from five $1 \mathrm{~s}$ samples (the radiometer time constant is $1 \mathrm{~s}$ ). Note the large brightness temperature difference between the triplesheet Mylar window and the "air" window and the small difference between the Teflon and foam window and the "air" window.

The column labeled "Measure" in Table II shows the experimental peak-to-peak and mean brightness temperature differences $\left(T_{d}\right)$ for the two cases. At $90 \mathrm{GHz}$, the Teflon and foam window produces much lower peak-to-peak and mean brightness temperature differences than the triple-sheet Mylar window. Note that the Teflon and foam window satisfies the brightness temperature variation criterion ( $T_{d}$ (peak to peak) $=0.5 \mathrm{~K})$ at $90 \mathrm{GHz}$, whereas the Mylar window does not $\left(T_{d}\right.$ (peak to peak) $=6.4 \mathrm{~K}$ ). The measured peak-to-peak values for 20 and $30 \mathrm{GHz}$ are not shown in Table II since the variation in air-gap sheet separation caused by shaking was not large enough to produce significant changes in interference at these wavelengths. Theoretical and experimental results for $T_{d}$ (mean) are in fair agreement with respect to frequency and window material (see Table II). Some of the difference between theoretical and experimental values for $T_{d}$ (mean) may be due to atmospheric brightness temperature fluctuations and assumptions used in the simplified theoretical model. A measured variation (peak to peak) of about $0.01 \mathrm{~mm}$ in apparent liquid water can be produced by shaking the triplesheet Mylar window. Measured variations (peak to peak) in water vapor for both cases and liquid water using the Teflon and foam window however are negligible; the values are below the noise level of the radiometric system.

\section{Application of the Results to Operational Radiometers}

Multifrequency radiometric instruments are becoming operational for continuous measurement of profiles of temperature and humidity under almost all weather conditions as well as for integrated water vapor and cloud liquid [11]. These instruments typically employ several channels in the 50-60 $\mathrm{GHz}$ range to profile temperature in addition to channels near 20 and $30 \mathrm{GHz}$. Typically, these systems employ an offset paraboloid antenna inside a building and an exposed $45^{\circ}$ flat reflector for directing the beam toward the zenith; between these two is a multisheet transmission window. We computed the performance of the two window designs in the $50-60 \mathrm{GHz}$ region using the analysis method outlined in Section III. Values of dielectric constant and loss tangent were estimated by extrapolating between the 31.6 and $90.0 \mathrm{GHz}$ values in Table I. The performance of the two types of window at the Profiler operating frequencies is summarized in Table IV. As expected, the theoretical brightness temperature fluctuations are much smaller with the Teflon and foam window than with the multisheet Mylar window: therefore, use of the former design would be expected to minimize errors in measurement of temperature and humidity profiles, and it is being used in "operational", Profiler systems.

\section{Conclusion}

The results presented here provide estimates of the effect of absorption and reflection by two types of multisheet transmission windows on atmospheric brightness temperature measurements. Both theory and experiment show that a Teflon and foam window causes less fluctuation and less difference in absolute value of brightness temperature than a triple-sheet Mylar window. With the Teflon and foam window, fluctuations at frequencies from 20 to $90 \mathrm{GHz}$ are at a level acceptable for maintaining radiometer accuracy. Furthermore, the Teflon and foam window produces much less noise than the triple-sheet Mylar window, thereby permitting precise measurements of integrated water vapor and cloud liquid.

Rigid fixed sheets would prevent sheet movement within a multisheet window and thus reduce the fluctuations in bright- 


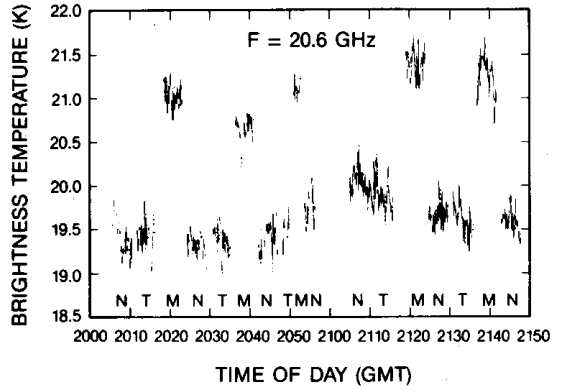

(a)

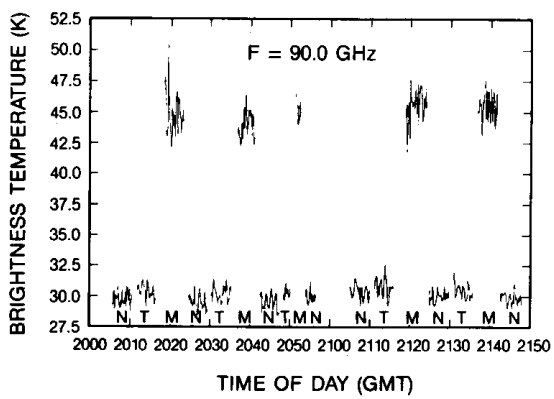

(c)

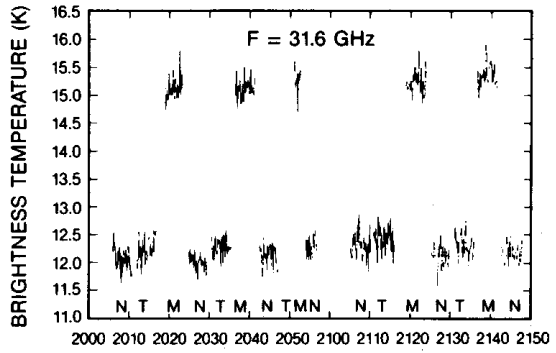

TIME OF DAY (GMT)

(b)

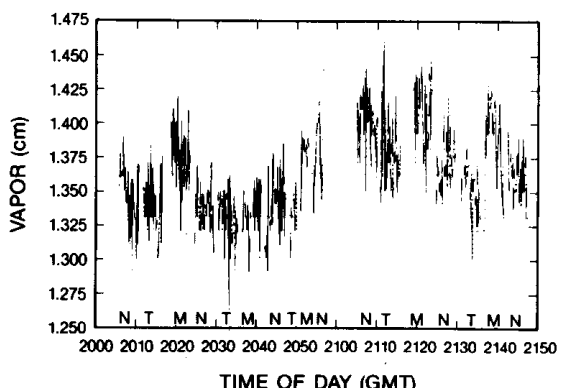

(d)

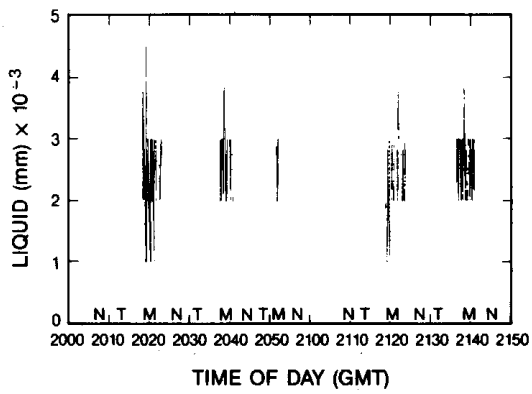

(e)

Fig. 5. Atmospheric brightness temperatures measured at (a) 20.6 , (b) 31.6 , (c) $90.0 \mathrm{GHz}$ with no window $(N)$, triple-sheet Mylar window $(M)$, and Teflon and foam window $(T)$. Apparent water vapor and liquid contents are shown in (d) and (e), respectively.

TABLE IV

COMPUTED BRIGHTNESS TEMPERATURES FOR THE TWO MULTISHEET WINDOWS AT CHANNELS IN THE 50-60 GHz RANGE $\left(T_{w}\right.$, AND $T$, ARE FIXED AT 298 AND $318 \mathrm{~K}$, RESPECTIVELY)

\begin{tabular}{|c|c|c|c|c|}
\hline \multirow[b]{2}{*}{$\begin{array}{l}\text { Frequency } \\
\qquad(\mathrm{GHz})\end{array}$} & \multicolumn{2}{|c|}{$\begin{array}{c}\text { Peak-to-Peak } \\
T_{d}\end{array}$} & \multicolumn{2}{|c|}{$\begin{array}{c}\text { Mean } \\
T_{d}\end{array}$} \\
\hline & $\begin{array}{c}\text { Mylar } \\
\text { (K) }\end{array}$ & $\begin{array}{l}\text { Teflon/foam } \\
\text { (K) }\end{array}$ & $\begin{array}{l}\text { Mylar } \\
\text { (K) }\end{array}$ & $\begin{array}{c}\text { Teflon/foam } \\
\text { (K) }\end{array}$ \\
\hline 52.9 & 4.0 & 0.2 & 2.3 & 0.2 \\
\hline 53.9 & 2.3 & 0.1 & 1.3 & 0.1 \\
\hline 55.5 & 0.9 & 0.1 & 0.5 & 0.1 \\
\hline 58.8 & 0.8 & 0.1 & 0.4 & 0.0 \\
\hline
\end{tabular}

ness temperature measurements, but they would probably be fairly thick, resulting in considerable emission. However, to the best of the authors' knowledge, no rigid sheet has been successfully implemented to give the low noise level required for sensitive radiometric measurements over a wide band of frequencies.

\section{ACKNOWLEDGMENT}

The authors thank James R. Jordan of the Wave Propagation Laboratory for useful discussions and help on the choice of the Teflon and foam window, the reviewers for their helpful comments, and Mildred Birchfield who expertly typed this manuscript.

\section{REFERENCES}

[1] D. C. Hogg, F. O. Guiraud, J. B. Snider, M. T. Decker, and E. R. Westwater, "A steerable dual-channel microwave radiometer for 
measurement of water vapor and liquid in the troposphere," J. Appl. Meteor., vol. 22, no. 5, pp. 789-806, May 1983.

[2] B. C. Blevis, "Losses due to rain on radomes and antenna reflecting surfaces," IEEE Trans. Antennas Propagat., vol. AP-13, pp. 175176, Jan. 1965.

[3] M. D. Jacobson, D. C. Hogg, and J. B. Snider, "Wet reflectors in millimeter-wave radiometry-Experiment and theory," IEEE Trans. Geosci. Remote Sensing, vol. GE-24, no. 5, pp. 784-791, 1986.

[4] C. T. A. Johnk, Engineering Electromagnetic Fields and Waves. New York: Wiley, pp. 493-497, 1975.

[5] K. C. Gupta, R. Garym, and R. Chadha, Computer-Aided Design of Microwave Circuits. Dedham, MA: Artech House, pp. 26-35, 1981

[6] A. R. Von Hippel, Tables of Dielectric Materials, Vol. V. Cambridge, MA: M.I.T. Laboratory for Insulation Research, 1957, p. 13.

[7] F. I. Shimabukuro, S. Lazar, M. R. Chernick, and H. G. Dyson, "A quasi-optical method for measuring the complex permittivity of material," IEEE Trans. Microwave Theory Technol., vol. MTT-32, no. 7, pp. 659-664, July 1984.

[8] R. G. Fellers, "Measurements at millimeter and micrometer wavelengths," Proc. IEEE, vol. 74, no. 1, pp. 36-38, 1986.

[9] M. T. Decker, E. R. Westwater, and F. O. Guiraud, "Experimental evaluation of ground-based microwave radiometeric sensing of atmospheric temperature and water vapor profiles," J. Appl. Meteorol., vol. 17 , pp. $1788-1795,1978$.

[10] E. R. Westwater, "The accuracy of water vapor and cloud liquid determination by dual-frequency ground-based microwave radiometry," Radio Sci., vol. 13, pp. 677-685, 1978.

[11] D. C. Hogg, M. T. Decker, F. O. Guirard, K. B. Earnshaw, D. W. van de Kamp, K. P. Moran, W. B. Sweezy, R. G. Strauch, E. R. Westwater, and C. G. Little, "An automatic profiler of the temperature, wind and humidity in the troposphere," J. Appl. Meteorol., vol. 22, no. 5, pp. 807-831, May 1983 .

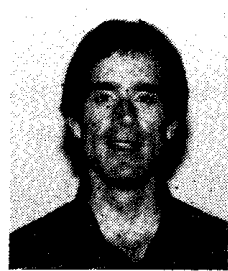

Mark D. Jacobson (S'79-M'80-S'82-M'85) was born in Billings, MT. He received the B.S.E.E. degree from Montana State University, Bozeman, in 1980, and the M.S.E.E. degree from the University of Colorado, Boulder, in 1985.

From 1980 to 1982 he was with IBM in Tucson, AZ, developing electronics for tape drives. He was a Teaching Assistant at the University of Colorado from 1982 to 1983 in the Electrical Engineering Department. From 1983 to 1984 he was employed part time at NOAA's Wave Propagation Laboratory in Boulder, $\mathrm{CO}$, where he was working on an antenna array for profiling the wind and involved in theoretical and experimental work on NOAA's dualchannel radiometers. From 1985 to 1986 he was with the Cooperative Institute for Research in Environmental Sciences, University of Colorado, in cooperation with NOAA's Wave Propagation Laboratory, where he worked on microwave radiometers. Since 1986 he has been with NOAA's Wave Propagation Laboratory in Boulder where he has been working on millimeter wave and infrared radiometers. His main technical interests are microwave and millimeter wave propagation, radiometry, and applied mathematics.

Mr. Jacobson is a member of Tau Beta Pi.

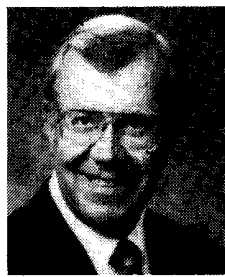

Jack B. Snider (M'61-M'66) received the B.S degree in electrical engineering from the University of Kansas, Lawrence, in 1957.

$\mathrm{He}$ is currently employed as a Supervisory Electronic Engineer by the Environmental Research Laboratories of the National Oceanic and Atmospheric Administration, Boulder, CO. Following three years with the Bendix Corporation, he joined the staff of the Central Radio Propagation Laboratory, National Bureau of Standards, in 1960 where he conducted several research projects in tropospheric radio propagation. He transferred to the Wave Propagation Laboratory in 1967. There his research has been concerned with the development of microwave and millimeter wave radiometric techniques for measurement of atmospheric absorption and for remote sensing of atmospheric temperature, water vapor, cloud liquid, and precipitation.

Mr. Snider is a member of Tau Beta Pi, Eta Kappa Nu, Commission F of the International Union for Radio Science, and the American Geophysical Union.

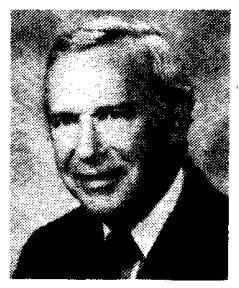

David C. Hogg (S'46-SM'57-F'65) received the B.Sc. degree in radio physics from the University of Western Ontario, London, Canada, in 1949, and the M.Sc. and Ph.D. degrees in physics from McGill University, Montreal, Canada, in 1951 and 1953, respectively.

From 1953 to 1966 he was a Member of Technical Staff in the Radio Research Department, from 1966 to 1973 Head of Atmospheric Research, and from 1973 to 1977 Head of Antennas and Propagation Research, at Bell Laboratories, Holmdel, NJ. He was Chief of Environmental Radiometry and Radio Meteorology at the Wave Propagation Laboratory, ERL, NOAA, Boulder, CO, from 1977 to 1986. At present he is with the Colorado Institute for Research in Environmental Sciences of the University of Colorado in Boulder. He has contributed to research in basic diffraction studies, microwave radio-relay systems, microwave antenna design, tropospheric beyond-horizon propagation, millimeter-wave propagation, in the initial low-noise experiments and sky-noise theory leading to the introduction of satellite communications utilizing the Echo and Telstar satellites and discovery of the microwave cosmic background noise, in some of the first work in visible and infrared propagation utilizing lasers, in design of the millimeter-wave beacons on the COMSTAR satellites, and in new techniques for remotely sensing the atmosphere. He and his colleagues established experimentally the differential attenuation and depolarization generated in transmission of microwaves through rain. His recent work includes design and construction of a Profiler System for automatic and continuous measurement of temperature, winds, and humidity in the troposphere, and development of microwave radiometric sensing systems for accurate measurement of water vapor, and liquid in clouds.

Dr. Hogg is a member of the National Academy of Engineering, a member and past United States Chairman of URSI Commission F, and a member AAAS. He is a recent recipient of a silver medal award by the United States Department of Commerce (1983) and of the Distinguished Achievement Award of the Geoscience and Remote Sensing Society of IEEE (1984). In 1985 he presented a series of lectures on Remote Sensing at Institutes in Japan and the People's Republic of China. 